\title{
Detection and Isolation of Simultaneous Additive and Parametric Faults in Nonlinear Stochastic Dynamical Systems
}

\author{
Andrés Cuervo, Pedro J. Zufiria, and Ángela Castillo \\ Departamento Matemática Aplicada a las Tecnologías de la Información, ETSI Telecomunicación, \\ Universidad Politécnica de Madrid (UPM), 28040 Madrid, Spain \\ Correspondence should be addressed to Pedro J. Zufiria, pedro.zufiria@upm.es
}

Received 31 August 2012; Revised 28 November 2012; Accepted 6 December 2012

Academic Editor: Huaguang Zhang

Copyright (C) 2012 Andrés Cuervo et al. This is an open access article distributed under the Creative Commons Attribution License, which permits unrestricted use, distribution, and reproduction in any medium, provided the original work is properly cited.

This paper presents a new fault detection and isolation scheme for dealing with simultaneous additive and parametric faults. The new design integrates a system for additive fault detection based on (Castillo and Zufiria, (2009)) and a new parametric fault detection and isolation scheme inspired in (Münz and Zufiria, (2008)) . It is shown that the so far existing schemes do not behave correctly when both additive and parametric faults occur simultaneously; to solve the problem a new integrated scheme is proposed. Computer simulation results are presented to confirm the theoretical studies.

\section{Introduction}

Motivated by the importance of safety in modern automated systems, fault detection and isolation schemes have received an increasing attention in the last two decades [1-4]. As opposed to costly hardware redundancy approaches, information redundancy schemes make use of data processing and system modelling paradigms, leading to either data-driven or model-based approaches. Among model-based fault diagnosis schemes, the FDI (Fault Detection and Isolation) techniques of the control community make use of explicit analytical models for redundancy checking [5].

The FDI analytical tools employed up to now can be classified into two main categories. On the one hand, stochastic discrete-time model-based schemes inherited from the signal estimation and linear control fields have successfully combined statistical schemes with geometrical tools in the design and characterization of detection algorithms for linear systems $[1-3,6]$. Nevertheless, these schemes have limited applicability since many real-world applications are grounded on the use of nonlinear models. On the 
other hand, deterministic continuous-time schemes coming from the adaptive and robust control community have proved to be suitable for nonlinear system modelling, where detection algorithms rely on the use of observer-type schemes to generate residuals whose profiles are evaluated [7-12]. In addition, some work has been performed in the design of accommodation schemes [13] or, more generally, Fault Tolerant Control (FTC) design [14, 15] that explicitly accounts for system nonlinearities and uncertainty $[16,17]$.

Recent research has also been focused on the design of diagnosis schemes for nonlinear stochastic systems, in order to cope with system and measurement noise. These schemes, such as the local approach [18], particle filters [19, 20], adaptive estimators [21], and hybrid system estimation based schemes [22], rely on discrete-time stochastic models, and they are also very computationally demanding, a major drawback for practical applications.

Alternatively, FDI schemes for continuous-time stochastic models have been recently developed [23-27], which are computationally less demanding. These schemes can be classified into two main categories: additive fault detectors [23, 24], and parametric fault detectors and isolators [25]; each of them is based on different techniques and assumptions. It is worth mentioning that further work has been carried out for implementing isolation schemes for additive faults [28], complementing the results in [24]. Concerning the FDI scheme for parametric faults in [25], it was valid for both detection and isolation. Although both types of schemes can be seen under a single unifying framework [26, 29], each of them was designed for addressing nonsimultaneous faults (either additive or multiplicative).

Complex real world systems are strongly interconnected, so that any subsystem failure can rapidly propagate abnormal behavior to other subsystems generating as a result new simultaneous failures [30-33]. Hence, additive and multiplicative faults are likely to occur simultaneously.

This paper presents a new detection and isolation scheme valid for simultaneous additive and parametric faults. The scheme makes use of improved versions of the methods proposed in [23-25]. Since detection of additive faults is not significantly affected by the presence of parametric faults, the work mainly focuses on the detection and isolation of parametric faults, which are more likely to provide specific information on the location of the system failure.

For doing so, we first show that the additive fault detection scheme proposed in [24] is robust against parametric faults; then, we illustrate the limitations of the parametric fault detection and isolation scheme proposed in [25] when additive faults are also present. Hence an improvement of this last scheme is proposed to overcome the problem.

The paper is organized as follows. In Section 2, the general framework for fault detection in nonlinear stochastic systems is presented. The existing schemes for detection and isolation of single faults are explained in Section 3, whereas the new proposed detection scheme is elaborated in Section 4. Section 5 illustrates the behavior of the presented scheme via simulation examples. Concluding remarks are summarized in Section 6.

\section{Problem Statement}

We consider the following class of nonlinear stochastic dynamical systems:

$$
\begin{gathered}
\dot{x}(t)=E_{n} x(t)+e_{n}\left(f\left(x(t), u(t), \vartheta_{0}, t\right)+\eta(t)+s\left(t-T_{0}\right) \phi(t)\right), \\
y(t)=h(x(t), u(t), t), \\
x(0)=x_{0},
\end{gathered}
$$


with

$$
E_{n}=\left[\begin{array}{ccccc}
0 & 1 & \cdots & \cdots & 0 \\
0 & 0 & 1 & \cdots & 0 \\
0 & 0 & \cdots & \ddots & \vdots \\
0 & 0 & 0 & \cdots & 1 \\
0 & 0 & 0 & \cdots & 0
\end{array}\right], \quad e_{n}=\left[\begin{array}{c}
0 \\
0 \\
\vdots \\
0 \\
1
\end{array}\right]
$$

which models, among other cases, any $n$th order nonlinear scalar system. Here, $x(t) \in \mathbb{R}^{n}$ is the system state, which has known initial value $x_{0} \in \mathbb{R}^{n} ; u(t) \in \mathbb{R}^{p}$ is the control input; the known function $f \in \mathbb{C}^{1}\left(\mathbb{R}^{n} \times \mathbb{R}^{p} \times \mathbb{R}^{m} \times \mathbb{R}^{+}, \mathbb{R}\right)$, which accordingly satisfies the Lipschitz condition, also satisfies that for all $x \in \mathbb{R}^{n}$ it holds $\|f(x, u, \vartheta, t)\| \leq C(1+\|x\|)$, for some constant $L$ and $C$, so that existence and uniqueness of solutions are guaranteed; $f$ represents the dynamics of the nominal model and has some parameters represented by $\vartheta_{0} \in \mathbb{R}^{m}$; the random vector $\eta: \mathbb{R}^{+} \rightarrow \mathbb{R}$, which gathers external disturbances and modelling errors, corresponds to a stochastic process of white Gaussian noise with autocorrelation function $R_{\eta}\left(t_{1}, t_{2}\right)=\sigma_{\eta} \delta\left(t_{1}-t_{2}\right)$ and noise intensity given by $\sigma_{\eta}$.

$y(t) \in \mathbb{R}^{l}$ is the measurable output, and the nonlinear mapping $h: \mathbb{R}^{n} \times \mathbb{R}^{p} \times \mathbb{R}^{+} \rightarrow \mathbb{R}^{l}$ can represent different output availability situations.

We assume that the pair $f, h$ allows the construction of an observer that provides $\tilde{x}$ as an accurate estimate of $x$, that is, sample-wise $\|\tilde{x}-x\| \leq \epsilon_{x}$; high gain observers [34, 35] and Lipschitz observers [36] have been successfully employed for this purpose. This paper mainly focused on the construction and the analysis of the so-called residual (to be explained in the following section) and addresses its estimation, the statistics of the estimator as well as the detectability and isolability conditions based on these statistics; hence, to simplify such exposition, an exact reconstruction of the state $x$ will be considered by assuming for the remainder of the paper that $\epsilon_{x}=0$ (i.e., $\tilde{x}=x$ ), which is a standard assumption for most nonlinear FDI schemes, as discussed in Section 1.

Finally, the fault function $\phi: \mathbb{R}^{+} \rightarrow \mathbb{R}$ can represent an unknown additive fault and/or a change in the parameters of the nominal part of the system, namely,

$$
\begin{aligned}
\phi(t) & =\phi_{a}(t)+\phi_{p}(t) \\
& =\phi_{a}(t)+\phi_{p}\left(x(t), u(t), \vartheta_{0}, \vartheta_{1}, t\right) \\
& =\phi_{a}(t)+f\left(x(t), u(t), \vartheta_{1}, t\right)-f\left(x(t), u(t), \vartheta_{0}, t\right) .
\end{aligned}
$$

Note that the possible simultaneous occurrence of both types of faults, generating complex $\phi(t)$ profiles, can make very difficult to unravel the fault origin.

The unit step function $s\left(t-T_{0}\right)$ is determined by $T_{0}$, the instant of time when the fault occurs. Note also that neither the postfailure parameter vector $\vartheta_{1}$ nor the time $T_{0}$ is known.

\subsection{Residual Construction}

Generally speaking, a residual is any variable whose behavior changes significantly when a fault occurs in the system. In this paper context, a (valid) residual will be a random variable (or stochastic process) whose statistical properties do change after a fault. 
Under the assumption of full-state availability we can create a new state variable $x_{c}(t)$ obtained from the following consistency equation:

$$
\dot{x}_{c}(t)=-\lambda\left(x_{c}(t)-x_{n}(t)\right)+f\left(x(t), u(t), \vartheta_{0}, t\right), \quad x_{c}(0)=x_{0},
$$

where $x_{c}(t) \in \mathbb{R}$ is the consistency checking state variable, and $\lambda>0$ is a design constant. Note that this equation makes use of the value of $x_{n}$, the $n$-th component of state variable $x$, in contrast to the estimated values usually employed in the design of observers. Subtracting (2.4) from system (2.1) we get a new variable $\epsilon(t)=x_{n}(t)-x_{c}(t)$ which depends on the model error and whose evolution is described by the following equation:

$$
\dot{\epsilon}(t)=-\lambda \epsilon(t)+\eta(t)+s\left(t-T_{0}\right) \phi(t), \quad \epsilon(0)=0 .
$$

The solution to this differential equation is

$$
\begin{aligned}
\epsilon(t) & =\int_{0}^{t} e^{-\lambda(t-\tau)} \eta(\tau) d \tau+\int_{0}^{t} e^{-\lambda(t-\tau)} s\left(\tau-T_{0}\right) \phi(\tau) d \tau \\
& =\epsilon_{\eta}(t)+\epsilon_{\phi}(t)
\end{aligned}
$$

where the model error $\epsilon(t)$ changes significantly after the occurrence of the fault $\left(t>T_{0}\right)$. Due to this property, the variable $\epsilon(t)$ has usually been utilized as the fundamental signal to construct valid residuals for detecting single faults. The algorithms for fault detection and isolation analyze the signal $\epsilon(t)$ by studying its statistical properties and its similarity with other reference signals. We will see that, when simultaneous faults do occur, $\epsilon(t)$ requires a more elaborated processing due to its potentially complex evolution.

\section{Single Fault Detection Schemes}

In this section, some existing schemes for the detection of single faults (either additive or parametric) are illustrated. The exposition is aimed to highlight those analytical aspects which will become relevant when designing the new improved scheme to be presented in Section 4.

\subsection{The Single Additive Fault Case}

The scheme in [24] analyzes the residual when $\phi(t)=\phi_{a}(t)$ and detects the additive faults under, roughly speaking, the unique condition that $E[\phi(t)]>\epsilon>0$ (or alternatively, $E[\phi(t)]<$ $\epsilon<0$ ) for all $t>T_{0}$ (see [24] for details). In addition, isolation schemes can be implemented assuming some conditions on the set of possible additive faults [28].

In general, these existing detection schemes will not be critically affected by the occurrence of a simultaneous parametric fault. Hence, we will see that the existing algorithms can be directly integrated into the new scheme proposed in Section 4. 


\subsection{Analysis of the Single Parametric Fault Case}

Under the assumption that a single parametric fault occurs, that is, $\phi(t)=\phi_{p}(t)$, this section presents the main results from [25], needed for the posterior analysis of the simultaneous fault case.

\subsubsection{Characterization of the Fault Function}

The scheme in [25] constructs a residual based on the signal $\epsilon_{\phi_{p}}$, using also the a priori knowledge about the fault function $\phi_{p}(t)$. The knowledge of such residual is limited due the unknown value of parameter $\vartheta_{1}$ as well as the unknown instant of time $T_{0}$.

In [25], a finite set of fault classes $\Theta$ is defined, and it is assumed that any faulty parameter vector $\vartheta_{1}$ belongs to one and just one of those classes. Furthermore, there exists a known function $\varphi\left(x, u, \vartheta_{0}, \Delta \vartheta, t\right)$ such that

$$
\phi_{p}\left(x, u, \vartheta_{0}, \vartheta_{1}, t\right)=k \varphi\left(x, u, \vartheta_{0}, \Delta \vartheta, t\right)
$$

where $\Delta \vartheta \in \mathbb{R}^{m}$ is a known vector specific of the fault class, and $k \in \mathbb{R}$ is an unknown constant that depends on which particular faulty parameter of the class has occurred. Note also that since the profile of $\varphi\left(x, u, \vartheta_{0}, \Delta \vartheta, t\right)$ depends on $x(t)$ it is also affected by $T_{0}$. This last dependence can be minimized by assuming that $T_{0}$ is large enough so that the system evolves within (or nearby) its $\omega$-limit set. Thus, a set of possible fault classes can be defined, and the fault function $\phi_{p}(t)$ will be approximately known for each $\vartheta_{1}$ except for a multiplicative constant $k$.

The fact that $T_{0}$ is unknown implies another limitation when computing the integrals; this fact leads to an approximation by defining

$$
\phi_{L P}(t)=\int_{0}^{t} e^{-\lambda(t-\tau)} \phi_{p}(\tau) d \tau=k \int_{0}^{t} e^{-\lambda(t-\tau)} \varphi_{p}(\tau) d \tau=k \varphi_{L P}(t)
$$

so that, for small parameter variations, the second summand of (2.6) satisfies $\epsilon_{\phi_{p}}(t)=$ $k \varphi_{L P}(t)-e^{-\lambda\left(t-T_{0}\right)} k \varphi_{L P}\left(T_{0}\right)$, where $\lim _{t \rightarrow \infty}\left(\epsilon_{\phi_{p}}(t)-k \varphi_{L P}(t)\right)=0$, meaning that

$$
\epsilon_{\phi_{p}}(t) \sim k \varphi_{L P}(t)
$$

As it will be shown in Section 4, alternative reference signals can be constructed to reduce the error associated with this approximation (3.3).

\subsubsection{Residual Generation}

After dealing with the unknown quantities, one can define the residual signal [25]:

$$
\cos \alpha_{\varphi \epsilon}(t)=\frac{\left\langle\epsilon, \varphi_{L P}\right\rangle_{T}}{\left\|\varphi_{L P}\right\|_{T}\|\epsilon\|_{T}}, \quad \text { with }\left\langle\epsilon, \varphi_{L P}\right\rangle_{T}=\frac{1}{T} \int_{t-T}^{t} E\left\{\epsilon(\tau) \varphi_{L P}(\tau)\right\} d \tau
$$


This residual, called moving angle, allows to formulate hypothesis test on it:

$$
\begin{gathered}
H_{0}: \cos \alpha_{\varphi \epsilon_{H_{0}}}(t)=\frac{\left\langle\varphi_{L P}, \epsilon_{H_{0}}\right\rangle_{T}(t)}{\left\|\varphi_{L P}\right\|_{T}(t)\left\|\epsilon_{H_{0}}\right\|_{T}(t)}=0 \\
H_{1}: \cos \alpha_{\varphi \epsilon_{H_{1}}}(t)= \\
\qquad \frac{\left\langle\varphi_{L P}, \epsilon_{H_{1}}\right\rangle_{T}(t)}{\left\|\varphi_{L P}\right\|_{T}(t)\left\|\epsilon_{H_{1}}\right\|_{T}(t)}=\frac{(1 / T) \int_{t-T}^{t} \varphi_{L P}(\tau) E\left[\epsilon_{H_{1}}(\tau)\right] d \tau}{\left\|\varphi_{L P}\right\|_{T}(t) \sqrt{(1 / T) \int_{t-T}^{t} E\left[\epsilon_{H_{1}}^{2}(\tau)\right] d \tau}} \\
\sim \frac{\left\|k \varphi_{L P}\right\|_{T}^{2}(t)}{\left\|k \varphi_{L P}\right\|_{T}(t) \sqrt{\left\|k \varphi_{L P}\right\|_{T}^{2}(t)+R_{\epsilon}}}=\frac{k}{\sqrt{k^{2}+R_{\epsilon} /\left\|\varphi_{L P}\right\|_{T}^{2}(t)}}
\end{gathered}
$$

where $\epsilon_{H_{0}}(t)=\epsilon_{\eta}(t)$ and $\epsilon_{H_{1}}(t)=\epsilon_{\eta}(t)+\epsilon_{\phi_{p}}(t)$. The moving angle changes significantly when there is a change in the system conditions from $H_{0}$ (no fault) to $H_{1}$ (fault), a behavior that corresponds to a good residual. In a practical application one can only calculate an estimation of the integral in (3.4)

$$
\left\langle\epsilon, \varphi_{L P}\right\rangle_{T, S}=\frac{1}{T} \int_{t-T}^{t} \epsilon(\tau) \varphi_{L P}(\tau) d \tau
$$

so the moving angle estimation is

$$
\cos \widehat{\alpha}_{\varphi \epsilon}(t)=\frac{\left\langle\epsilon, \varphi_{L P}\right\rangle_{T, S}}{\left\|\varphi_{L P}\right\|_{T, S}\|\epsilon\|_{T, S}} .
$$

Note that such estimator is defined by a quotient of the form $g=X / \sqrt{Y}$, where $X$ and $Y$ are random variables; hence its expected value and variance can be computed upon [37]

$$
\begin{gathered}
E[g] \sim \frac{E[X]}{\sqrt{E[Y]}}-\frac{\operatorname{Cor}[X, Y]}{2 \sqrt{E[Y]^{3}}}+\frac{3 E[X] \operatorname{Var}[Y]}{8 \sqrt{E[Y]^{5}}}, \\
\operatorname{Var}[g] \sim \frac{\operatorname{Var}[X]}{E[Y]}-\frac{\operatorname{Cor}[X, Y] E[X]}{E[Y]^{2}}+\frac{E[X]^{2} \operatorname{Var}[Y]}{4 E[Y]^{3}} .
\end{gathered}
$$

Applying this result to (3.7), with $\left\|\varphi_{L P}\right\|_{T, S}$ deterministic and $X=\left\langle\varphi_{L P}, \epsilon\right\rangle_{T, S}$, $Y=\|\epsilon\|_{T, S}^{2}$, we obtain the expressions shown in Table 1 (where $\operatorname{Var}\left[X \mid H_{0}\right]=$ $\left.\left(1 / T^{2}\right) \int_{t-T}^{t} \int_{t-T}^{t} \varphi_{L P}\left(\tau_{1}\right) \varphi_{L P}\left(\tau_{2}\right) R_{\epsilon}\left(\tau_{1}, \tau_{2}\right) d \tau_{1} d \tau_{2}\right)$. 
Then, the resulting expressions for the estimator moments under the different hypotheses are

$$
\begin{gathered}
E\left[\cos \widehat{\alpha}_{\varphi \tilde{\epsilon}_{H_{0}}}\right]=0, \\
E\left[\cos \widehat{\alpha}_{\left.\varphi_{L P} \tilde{e}_{H_{1}}\right]}\right] \frac{\operatorname{Var}\left[\cos \widehat{\alpha}_{\varphi \tilde{\epsilon}_{H_{0}}}\right]=\frac{\operatorname{Var}\left[X \mid H_{0}\right]}{\left\|\varphi_{L P}\right\|_{T}^{2} R_{\epsilon}},}{\left\|\varphi_{L P}\right\|_{T} \sqrt{R_{\epsilon}+k^{2}\left\|\varphi_{L P}\right\|_{T}^{2}}} \\
\times\left(k\left\|\varphi_{L P}\right\|_{T}^{2}-\frac{2 k \operatorname{Var}\left[X \mid H_{0}\right]}{2\left(R_{\epsilon}+k^{2}\left\|\varphi_{L P}\right\|_{T}^{2}\right)}+\right. \\
\left.+\frac{3 k\left\|\varphi_{L P}\right\|_{T}^{2}\left(\left(R_{\epsilon}^{2} / \lambda^{2} T^{2}\right)(2 \lambda T-1)+4 k^{2} \operatorname{Var}\left[X \mid H_{0}\right]\right)}{8\left(R_{\epsilon}+k^{2}\left\|\varphi_{L P}\right\|_{T}^{2}\right)^{2}}\right)
\end{gathered}
$$

$$
\begin{aligned}
& \operatorname{Var}\left[\cos \widehat{\alpha}_{\varphi \tilde{\epsilon}_{H_{1}}}\right] \sim \frac{1}{\left\|\varphi_{L P}\right\|_{T}\left(R_{\epsilon}+k^{2}\left\|\varphi_{L P}\right\|_{T}^{2}\right)} \\
& \times\left(\operatorname{Var}\left[X \mid H_{0}\right]-\frac{2 k^{3}\left\|\varphi_{L P}\right\|_{T}^{2} \operatorname{Var}\left[X \mid H_{0}\right]}{\left(R_{\epsilon}+k^{2}\left\|\varphi_{L P}\right\|_{T}^{2}\right)}\right. \\
& \left.+\frac{k^{2}\left\|\varphi_{L P}\right\|_{T}^{4}\left(\left(R_{\epsilon}^{2} / \lambda^{2} T^{2}\right)(2 \lambda T-1)+4 k^{2} \operatorname{Var}\left[X \mid H_{0}\right]\right)}{4\left(R_{\epsilon}+k^{2}\left\|\varphi_{L P}\right\|_{T}^{2}\right)^{2}}\right) .
\end{aligned}
$$

Based on these deterministic quantities we can construct $\gamma$ confidence intervals of $\cos \widehat{\alpha}_{\varphi \tilde{\varepsilon}}(t)$ under both hypotheses $H_{0}$ and $H_{1}$ :

$$
\begin{gathered}
\Delta_{\cos \widehat{\alpha}_{\varphi e_{H_{0}}}}=\left[\cos \underline{\alpha}_{\varphi \epsilon_{H_{0}}}, \cos \bar{\alpha}_{\varphi \epsilon_{H_{0}}}\right], \\
\Delta_{\cos \widehat{\alpha}_{\varphi e_{H_{1}}}}=\left[\cos \underline{\alpha}_{\varphi \epsilon_{H_{1}}}, \cos \bar{\alpha}_{\varphi \epsilon_{H_{1}}}\right], \\
\Delta_{\cos \hat{\alpha}_{\varphi e_{H_{0}}}} \cap \Delta_{\cos \hat{\alpha}_{\varphi e_{H_{1}}}}=\emptyset
\end{gathered}
$$

where

$$
\begin{aligned}
& \cos \underline{\alpha}_{\varphi \epsilon_{H_{0}}} \approx E\left[\cos \widehat{\alpha}_{\varphi \epsilon_{H_{0}}}\right]-h_{\gamma / 2} \sqrt{\operatorname{Var}\left[\cos \widehat{\alpha}_{\varphi \epsilon_{H_{0}}}\right]}, \\
& \cos \bar{\alpha}_{\varphi \epsilon_{H_{0}}} \approx E\left[\cos \widehat{\alpha}_{\varphi \epsilon_{H_{0}}}\right]+h_{\gamma / 2} \sqrt{\operatorname{Var}\left[\cos \widehat{\alpha}_{\varphi \epsilon_{H_{0}}}\right]}, \\
& \cos \underline{\alpha}_{\varphi \epsilon_{H_{1}}} \approx E\left[\cos \widehat{\alpha}_{\varphi \epsilon_{H_{1}}}\right]-h_{\gamma / 2} \sqrt{\operatorname{Var}\left[\cos \widehat{\alpha}_{\varphi \epsilon_{H_{1}}}\right]}, \\
& \cos \bar{\alpha}_{\varphi \epsilon_{H_{1}}} \approx E\left[\cos \widehat{\alpha}_{\varphi \epsilon_{H_{1}}}\right]+h_{\gamma / 2} \sqrt{\operatorname{Var}\left[\cos \widehat{\alpha}_{\varphi \epsilon_{H_{1}}}\right]} .
\end{aligned}
$$


These confidence intervals ensure that the estimator will take values on each one of them with probability $r$ when the system is operating under the corresponding hypotheses. The detection scheme is triggered when the residual estimator enters the interval corresponding to $H_{1}$ (see [25] for details).

\section{The New Simultaneous Fault Detection and Isolation Scheme}

When simultaneous faults occur, they may disguise each other's effects, increasing the difficulty of their detection; in such case, existing schemes for a separate fault detection may not work. In this Section, the simultaneous fault case is considered, and a new scheme for addressing this problem is proposed. The proposed detection scheme integrates improved versions of the algorithms proposed in [24] for additive faults and the one presented in [25] for parametric ones.

\subsection{Analysis of the Simultaneous Fault Situation}

As mentioned in Section 1, simultaneous faults are likely to occur in real-world systems. Nevertheless, most standard FDI schemes assume that only one single fault occurs at a time. In some specific cases, separation mechanisms have been developed [9], which are not directly applicable in general. Here, we analyze the schemes presented in [24, 25] under simultaneous additive and parametric faults.

If a parametric and an additive fault occur at the same time (we label this hypothesis of simultaneous faults as $\left.H_{1}^{\text {sim }}\right)$, (2.6) becomes

$$
\dot{\epsilon}(t)=-\lambda \epsilon(t)+\eta(t)+s\left(t-T_{0}\right)\left(\phi_{p}(t)+\phi_{a}(t)\right)
$$

where $\phi_{p}(t)$ is the parametric fault function 2 and $\phi_{a}(t)$ is a stochastic process with constant

mean $E\left[\phi_{a}(t)\right]=\bar{\phi}_{a^{\prime}}, \forall t$. The solution of this stochastic differential equation has three summands

$$
\epsilon_{H_{1}^{\mathrm{sim}}}(t)=\epsilon_{H_{0}}(t)+\epsilon_{\phi_{a}}(t)+\epsilon_{\phi_{p}}(t)
$$

\subsection{Additive Fault Detection Scheme}

As mentioned above, the scheme in [24] analyzes the residual $\epsilon(t)$ and detects the additive faults under, roughly speaking, the unique condition that $E[\phi(t)]>\epsilon>0$ (or alternatively, $E[\phi(t)]<\epsilon<0)$ for all $t>T_{0}$. In general, when $\phi_{a}(t)$ satisfies the detectability condition, such that $\left|E\left[\phi_{a}(t)\right]\right|=\left|\bar{\phi}_{a}\right|>\epsilon$, it is very unlikely that a parametric fault would generate a significant value of $E\left[\phi_{p}(t)\right]$ that would precisely compensate and mask the additive term. In practice, the errors caused by (initially small) parameter variations imply that $E\left[\phi_{p}(t)\right] \approx 0$, so that $E[\phi(t)] \approx E\left[\phi_{a}(t)\right]$, and the additive fault detection scheme will not be affected by such simultaneous parametric faults.

The main challenge then becomes to detect and isolate the parametric faults in such working environment $\left(E\left[\phi_{p}(t)\right] \approx 0\right)$. Interestingly, the profile of $\phi_{p}(t)$ may allow for the fault detection and isolation, as shown below. 
Table 1: Statistics of magnitudes $X$ and $Y$ under the $H_{0}$ and $H_{1}$ hypotheses.

\begin{tabular}{lcc}
\hline Hypothesis & $H_{0}$ & $H_{1}$ \\
\hline$E[X]$ & 0 & $k\left\|\varphi_{L P}\right\|_{T, S}^{2}$ \\
$\operatorname{Var}[X]$ & $\operatorname{Var}\left[X \mid H_{0}\right]$ & $\operatorname{Var}\left[X \mid H_{0}\right]$ \\
$E[Y]$ & $R_{\epsilon}$ & $k^{2}\left\|\varphi_{L P}\right\|_{T, S}^{2}+R_{\epsilon}$ \\
$\operatorname{Var}[Y]$ & $\frac{R_{\epsilon}^{2}}{\lambda^{2} T^{2}}(2 \lambda T-1)$ & $\frac{R_{\epsilon}^{2}}{\lambda^{2} T^{2}}(2 \lambda T-1)+4 k^{2} \operatorname{Var}\left[X \mid H_{0}\right]$ \\
$\operatorname{Cor}[X, Y]$ & 0 & $2 k \operatorname{Var}\left[X \mid H_{0}\right]$ \\
\hline
\end{tabular}

\subsection{Parametric Fault Residuals for Simultaneous Case}

As it is shown below, the parametric fault detection and isolation scheme presented in [25] are likely to be disturbed by the occurrence of simultaneous additive faults disguising parametric faults. In the following section we modify such scheme in order to reduce its sensitivity to these additive faults.

Assuming that the extra summand asymptotically behaves

$$
\begin{aligned}
\epsilon_{\phi_{a}}(t) & =\int_{0}^{t} e^{-\lambda(t-\tau)} s\left(\tau-T_{0}\right) \phi_{a}(\tau) d \tau=\frac{\bar{\phi}_{a} e^{\lambda-t}}{\lambda}\left[e^{\lambda t}\right]_{T_{0}}^{t} \\
& =\frac{\bar{\phi}_{a}}{\lambda}\left(1-e^{\lambda\left(T_{0}-t\right)}\right) \sim \frac{\bar{\phi}_{a}}{\lambda}=k_{\phi_{a}} .
\end{aligned}
$$

So the model error under hypothesis $H_{1}^{\text {sim }}$ tends to

$$
\epsilon_{H_{1}^{\text {sim }}}(t) \sim \epsilon_{H_{0}}(t)+k_{\phi_{a}}+k \varphi_{L P}(t)
$$

Hence, the moving angle takes the following asymptotic expression:

$$
\cos \alpha_{\varphi \epsilon_{H_{1}^{\text {sim }}}} \sim \frac{\left\langle\epsilon_{H_{0}}+k_{\phi_{a}}+k \varphi_{L P}, \varphi_{L P}\right\rangle}{\left\|\varphi_{L P}\right\|_{T} \sqrt{\left\langle\epsilon_{H_{0}}+k_{\phi_{a}}+k \varphi_{L P}, \epsilon_{H_{0}}+k_{\phi_{a}}+k \varphi_{L P}\right\rangle}} .
$$

We observe that the additive fault affects both the numerator and the denominator of the moving angle. Once again this quantity has to be estimated, and its statistics are calculated. The components of the expressions of the expected value and the variance are shown in Table 2 (where $\bar{\varphi}_{L P}=(1 / T) \int_{t-T}^{t} \varphi_{L P}(\tau) d \tau$ and $\left.V_{\varphi \epsilon}=\left(1 / T^{2}\right) \iint_{t-T}^{t} \varphi_{L P}\left(\tau_{1}\right) R_{\epsilon}\left(\tau_{1}, \tau_{2}\right) d \tau_{1} d \tau_{2}\right)$.

When compared to Table 1 , several new additive terms show up in Table 2 . This fact limits the performance of the estimator under hypotheses $H_{1}^{\text {sim }}$ as compared to the case $H_{1}^{p}$ (single parametric fault); fortunately, some approximations can be made. In fact, under the hypothesis of small parameter variation $\left(E\left[\phi_{p}(t)\right] \approx 0\right)$, we have that even if $\varphi_{L P}(t)$ might oscillate, the ergodicity assumption justifies that $\bar{\varphi}_{L P}$ evolves in a smaller range so that the corresponding terms can be neglected; hence, the most significant term is $k_{\phi_{a}}^{2}$, due to the additive fault, so that Table 2 can be simplified to Table 3 . 
Table 2: Statistics of magnitudes $X$ and $Y$ under the $H_{1}^{\text {sim }}$ hypotheses.

\begin{tabular}{ll}
\hline Hypothesis & $H_{1}^{\text {sim }}$ \\
\hline$E[X]$ & $k_{\phi_{a}} \bar{\varphi}_{L P}+k\left\|\varphi_{L P}\right\|_{T}^{2}$ \\
$\operatorname{Var}[X]$ & $\operatorname{Var}\left[X \mid H_{0}\right]$ \\
$E[Y]$ & $R_{e}+k_{\phi_{a}}^{2}+k^{2}\left\|\varphi_{L P}\right\|_{T}^{2}+2 k k_{\phi_{a}} \bar{\varphi}_{L P}$ \\
$\operatorname{Var}[Y]$ & $\frac{R_{\epsilon}^{2}}{\lambda^{2} T^{2}}(2 \lambda T-1)+4 k^{2} \operatorname{Var}\left[X \mid H_{0}\right]+8 k k_{\phi_{a}} V_{\varphi e}+\frac{8 k_{\phi_{a}}^{2} R_{\epsilon}}{T^{2} \lambda^{2}}\left(\frac{-T^{2} \lambda^{2}}{4}+\lambda T-1\right)$ \\
$\operatorname{Cor}[X, Y]$ & $2 k \operatorname{Var}\left[X \mid H_{0}\right]+2 k_{\phi_{a}} V_{\varphi \epsilon}$ \\
\hline
\end{tabular}

Table 3: Simplified statistics of magnitudes $X$ and $Y$ under the $H_{1}^{\text {sim }}$ hypotheses.

\begin{tabular}{ll}
\hline Hypothesis & $H_{1}^{\text {sim }}$ \\
\hline$E[X]$ & $k\left\|\varphi_{L P}\right\|_{T}^{2}$ \\
$\operatorname{Var}[X]$ & $\operatorname{Var}\left[X \mid H_{0}\right]$ \\
$E[Y]$ & $R_{\epsilon}+k^{2}\left\|\varphi_{L P}\right\|_{T}^{2}+k_{\phi_{a}}^{2}$ \\
$\operatorname{Var}[Y]$ & $\frac{R_{\epsilon}^{2}}{\lambda^{2} T^{2}}(2 \lambda T-1)+4 k^{2} \operatorname{Var}\left[X \mid H_{0}\right]$ \\
$\operatorname{Cor}[X, Y]$ & $2 k \operatorname{Var}\left[X \mid H_{0}\right]$ \\
\hline
\end{tabular}

Using this approximation, the expected value and variance of the estimator under hypothesis $H_{1}^{\text {sim }}$ are

$$
\begin{aligned}
& E\left[\cos \widehat{\alpha}_{\varphi \widetilde{\epsilon}_{H_{1}^{s i m}}}\right] \\
& \sim \frac{1}{\left\|\varphi_{L P}\right\|_{T} \sqrt{R_{\epsilon}+k^{2}\left\|\varphi_{L P}\right\|_{T}^{2}+k_{\phi_{a}}^{2}}} \\
& \times\left(k\left\|\varphi_{L P}\right\|_{T}^{2}-\frac{2 k \operatorname{Var}\left[X \mid H_{0}\right]}{2\left(R_{\epsilon}+k^{2}\left\|\varphi_{L P}\right\|_{T}^{2}+k_{\phi_{a}}^{2}\right)}\right. \\
& \left.+\frac{3 k\left\|\varphi_{L P}\right\|_{T}^{2}\left(\left(R_{\epsilon}^{2} / \lambda^{2} T^{2}\right)(2 \lambda T-1)+4 k^{2} \operatorname{Var}\left[X \mid H_{0}\right]+\left(8 k_{\phi_{a}}^{2} R_{\epsilon} / T^{2} \lambda^{2}\right)\left(-T^{2} \lambda^{2} / 4+\lambda T-1\right)\right)}{8\left(R_{\epsilon}+k^{2}\left\|\varphi_{L P}\right\|_{T}^{2}+k_{\phi_{a}}^{2}\right)^{2}}\right), \\
& \operatorname{Var}\left[\cos \widehat{\alpha}_{\varphi \tilde{\varepsilon}_{H_{1}^{\operatorname{sim}}}}\right] \\
& \sim \frac{1}{\left\|\varphi_{L P}\right\|_{T}\left(R_{\epsilon}+k^{2}\left\|\varphi_{L P}\right\|_{T}^{2}+k_{\phi_{a}}^{2}\right)} \\
& \times\left(\operatorname{Var}\left[X \mid H_{0}\right]-\frac{2 k^{3}\left\|\varphi_{L P}\right\|_{T}^{2} \operatorname{Var}\left[X \mid H_{0}\right]}{\left(R_{\epsilon}+k^{2}\left\|\varphi_{L P}\right\|_{T}^{2}+k_{\phi_{a}}^{2}\right)}\right. \\
& \left.+\frac{k^{2}\left\|\varphi_{L P}\right\|_{T}^{4}\left(\left(R_{\epsilon}^{2} / \lambda^{2} T^{2}\right)(2 \lambda T-1)+4 k^{2} \operatorname{Var}\left[X \mid H_{0}\right]+\left(8 k_{\phi_{a}}^{2} R_{\epsilon} / T^{2} \lambda^{2}\right)\left(-T^{2} \lambda^{2} / 4+\lambda T-1\right)\right)}{4\left(R_{\epsilon}+k^{2}\left\|\varphi_{L P}\right\|_{T}^{2}+k_{\phi_{a}}^{2}\right)^{2}}\right) .
\end{aligned}
$$


Both these quantities are considerably different compared to their counterparts under hypothesis $H_{1}^{p}$. Thus, the $\gamma$ confidence interval under hypotheses $H_{1}^{\text {sim }}$ and $H_{1}^{p}$ verifies $\Delta_{\cos \widehat{\alpha}_{\varphi e_{H}} H_{1}^{\text {sim }}} \neq \Delta_{\cos \widehat{\alpha}_{\varphi e_{H_{1}^{p}}^{p}}}$. This fact causes several detection problems, since the detection scheme checks if the estimator belongs to $\Delta_{\cos \widehat{\alpha}_{\varphi e} H_{1}^{p}}$ to trigger the alarm; however, under the $H_{1}^{\text {sim }}$ hypothesis it will belong to $\Delta_{\cos \widehat{\alpha}_{\varphi e^{\text {sim }}}^{\text {sim }}}$ with probability $\gamma$.

\subsection{Improvements on the Detection Scheme}

The scheme presented in [25] has been improved in two directions. On the one hand, the reference signals have been obtained in a way that reduces the error associated with the approximation in (3.3); on the other hand, the influence of the additive error has been minimized via an appropriate filtering of $\epsilon(t)$.

\subsubsection{Improving the Reference Signal}

The reference signal proposed in [25]

$$
\phi_{L P}(t)=\int_{0}^{t} e^{-\lambda(t-\tau)} \phi_{p}(\tau) d \tau
$$

is computed integrated from the 0 initial time, since the real value of $T_{0}$ is unknown. Nevertheless, it is possible to define a new reference signal

$$
\phi_{L P, \underline{T_{0}}}(t)=\int_{\underline{T_{0}}}^{t} e^{-\lambda(t-\tau)} \phi_{p}(\tau) d \tau,
$$

where $T_{0}$ can be dynamically chosen, for instance, as $\underline{T_{0}}=t-T$ the lower bound of the interval $[t-T, t]$ where the moving angle is defined. The value of $\underline{T_{0}}$ is likely to be closer to $T_{0}$ than the 0 value. Hence,

$$
\epsilon_{\phi_{p}}(t)=k \varphi_{L P, T_{0}}(t)-e^{-\lambda\left(t-T_{0}\right)} k \varphi_{L P, \underline{T_{0}}}\left(T_{0}\right),
$$

and, if the faults occur in the interval $[t-T, t] \ni T_{0}$, then $\left|T_{0}-T_{0}\right|<T$, and we obtain the bound

$$
\left|\varphi_{L P, T_{\underline{T}}}\left(T_{0}\right)\right| \leq\left\|\varphi_{p}(\tau)\right\|_{[t-T, t]}^{\infty} \cdot T
$$

so that the term $e^{-\lambda\left(t-T_{0}\right)} k \varphi_{L P, T_{0}}\left(T_{0}\right)$ will be small. This means that the new approximation

$$
\epsilon_{\phi_{p}}(t) \sim k \varphi_{L P, T_{0}}(t)
$$

will have, in general, a smaller error than (3.3); this fact justifies the good performance of the newly proposed reference signals. 


\subsubsection{Eliminating the Additive Term Influence}

The analysis in Section 4.3 shows that the detection scheme presented in [25] does not work correctly under hypothesis $H_{1}^{\text {sim }}$ because of the influence of $k_{\phi_{a}}^{2}$. Note that equality (4.4) demonstrates that asymptotically $k_{\phi_{a}}$ is a constant term added to $\epsilon(t)$. Thus, one way to vanish its effect is to low pass filter $\epsilon(t)$. Let $\widehat{\epsilon}(t)$ be a filtered version of $\epsilon(t)$ :

$$
\widehat{\epsilon}(t)=\epsilon(t)-\frac{1}{T} \int_{t-T}^{t} \epsilon(\tau) d \tau
$$

In this case, under hypothesis $H_{1}^{\text {sim }}$ we have

$$
\begin{aligned}
\widehat{\epsilon}_{H_{1}^{\text {sim }}}(t) & =\epsilon_{H_{0}}(t)+\epsilon_{\phi_{a}}(t)+k \varphi_{L P}(t)-\frac{1}{T} \int_{t-T}^{t} \epsilon_{H_{0}}(\tau) d \tau-\frac{1}{T} \int_{t-T}^{t} \epsilon_{\phi_{a}}(\tau) d \tau-\frac{1}{T} \int_{t-T}^{t} \varphi_{L P}(\tau) d \tau \\
& =\epsilon_{H_{0}}(t)+k \varphi_{L P}(t)-\bar{\epsilon}_{H_{0}}(t)-\bar{\varphi}_{L P}(t) .
\end{aligned}
$$

Since $E\left[\epsilon_{H_{0}}(t)\right]=0$, the ergodicity assumption allows us to consider $\bar{\epsilon}_{H_{0}}(t) \approx 0$. Hence, the statistics of the estimator of the moving angle are now calculated using the elements of Table 4, where

$$
\begin{aligned}
& V_{\bar{\varphi}^{2} \epsilon}=\frac{1}{T^{2}} \iint_{t-T}^{t} \bar{\varphi}_{L P}\left(\tau_{1}\right) \bar{\varphi}_{L P}\left(\tau_{2}\right) R_{\epsilon}\left(\tau_{1}, \tau_{2}\right) d \tau_{1} d \tau_{2}, \\
& V_{\varphi \bar{\varphi} \epsilon}=\frac{1}{T^{2}} \iint_{t-T}^{t} \varphi_{L P}\left(\tau_{1}\right) \bar{\varphi}_{L P}\left(\tau_{2}\right) R_{\epsilon}\left(\tau_{1}, \tau_{2}\right) d \tau_{1} d \tau_{2} .
\end{aligned}
$$

Comparing this table to Table 2 , one can see that the term $k_{\phi_{a}}$ does not show up in any term. Finally, under the usual conditions mentioned in Section $4.2\left(E\left[\phi_{p}(t) \approx 0\right.\right.$, and ergodicity), we have that $\bar{\varphi}_{L P} \approx 0$, so that the terms involving $\bar{\varphi}_{L P}$ (i.e., $\left\langle\bar{\varphi}_{L P}, \bar{\varphi}_{L P}\right\rangle,\left\langle\varphi_{L P}, \bar{\varphi}_{L P}\right\rangle, V_{\bar{\varphi}^{2} \epsilon^{\prime}}$ and $V_{\varphi \bar{\varphi} \epsilon}$ ) are negligible. Hence, the expected value and the variance of the moving angle satisfy

$$
\begin{aligned}
E\left[\cos \alpha_{\varphi \epsilon_{H_{1}^{p}}}\right] & \approx E\left[\cos \alpha_{\varphi \epsilon_{H_{1}^{\text {sim }}}}\right], \\
\operatorname{Var}\left[\cos \alpha_{\varphi \epsilon_{H_{1}^{p}}}\right] & \approx \operatorname{Var}\left[\cos \alpha_{\varphi \epsilon_{H_{1}^{\text {sim }}}}\right],
\end{aligned}
$$

meaning that the new detection and isolation procedures proposed here can be successfully applied.

It is worth mentioning that the new resulting scheme is applicable to simultaneous faults composed by additive and parametric faults that satisfy similar detectability and isolability conditions to the ones stated in [24, 25], respectively. Concerning detection and isolation times, although the filtering process may slightly delay the responses, in general the detection and isolation times are similar to the original schemes times, as shown in the following example. 
Table 4: Statistics of the magnitudes $X$ and $Y$ under the $H_{1}^{\text {sim }}$ hypotheses after the filtering process.

\begin{tabular}{ll}
\hline Hypothesis & $H_{1}^{\text {sim }}$ \\
\hline$E[X]$ & $k\left\|\varphi_{L P}\right\|_{T}^{2}-\left\langle\varphi_{L P}, \bar{\varphi}_{L P}\right\rangle$ \\
$\operatorname{Var}[X]$ & $\operatorname{Var}\left[X \mid H_{0}\right]$ \\
$E[Y]$ & $R_{e}+k^{2}\left\|\varphi_{L P}\right\|_{T}^{2}+\left\langle\bar{\varphi}_{L P}, \bar{\varphi}_{L P}\right\rangle-2 k\left\langle\varphi_{L P}, \bar{\varphi}_{L P}\right\rangle$ \\
$\operatorname{Var}[Y]$ & $\frac{R_{\epsilon}^{2}}{\lambda^{2} T^{2}}(2 \lambda T-1)+4 k^{2} \operatorname{Var}\left[X \mid H_{0}\right]+4 V_{\bar{\varphi}^{2} \epsilon}-8 k V_{\varphi \bar{\varphi} e}$ \\
$\operatorname{Cor}[X, Y]$ & $2 k \operatorname{Var}\left[X \mid H_{0}\right]-2 V_{\varphi \bar{\varphi} e}$ \\
\hline
\end{tabular}

Finally, note that such detection and isolation times do have a clear impact on the fault accommodation strategy to be applied [13].

\section{Application Example}

\subsection{Simulation Setup}

Here the correct behavior of the work presented in the previous sections is illustrated with the Van der Pol oscillator $(\mathrm{VdPO}) \ddot{y}+2 \omega \zeta\left(\mu y^{2}-1\right) \dot{y}+\omega^{2} y=0$ via simulations with Matlab Simulink. The election of this system has also been made in other works on deterministic system fault diagnosis [38] as well as in the study of stochastic systems [24, 25] as it is the case here.

The VdPO describes an LC oscillator with nonlinear resistive element such as a tunnel diode. The output $y$ represents the voltage at the inductor, whereas $\dot{y}$ is the current through this inductor. In this simulation, it is considered that all electrical elements are not ideal (e.g., due to change of temperature) but stochastically varying. Consequently, we obtain the following state space representation of the VdPO:

$$
\begin{gathered}
\dot{x}_{1}=x_{2}(t)+\eta_{1}(t) \\
\dot{x}_{2}=2 \omega \zeta\left(1-\mu x_{1}^{2}\right) x_{2}-\omega^{2} x_{1}+\eta_{2}(t),
\end{gathered}
$$

where $\eta_{i}, i=1,2$ are normalized white Gaussian noise with zero mean and auto correlation $R_{\eta_{i}}\left(t_{1}, t_{2}\right)=\delta\left(t_{1}-t_{2}\right)$. We assume that both states are measurable as indicated in Section 2 . The system function is $f(x, u, \theta, t)=2 \omega \zeta\left(1-\mu x_{1}^{2}\right) x_{2}-\omega^{2} x_{1}$ with $\theta=[\omega, \zeta, \mu]^{T}$.

This system presents a nice feature: it is linear in $\zeta$ and $\mu$ and nonlinear in $\omega$. Hence, fault functions that are both linear and nonlinear in $\Delta \theta$ can be investigated; in this example we focus on the detection of faults on the nonlinear parameter, $\omega$. Moreover, the oscillator runs on stable limit cycles for $\omega, \zeta, \mu>0$, which do change slightly for small parameter changes. Despite this fact, the detection scheme presented in [25] successfully detects these single faults.

A fault class is defined for the $\omega$ parameter whose corresponding representative is

$$
\varphi_{w}\left(x, u, \theta_{0}, \Delta \omega, t\right)=\Delta \omega\left(2 \zeta_{0}\left(1-\mu_{0} x_{1}^{2}\right) x_{2}-2 \omega_{0} x_{1}\right) .
$$


Table 5: Relevant simulation parameters.

\begin{tabular}{lc}
\hline Parameter & Value \\
\hline$T$ & 10 \\
$\lambda$ & 25 \\
$T_{0}$ & 15 \\
$\omega_{0}$ & 1 \\
$\Delta \omega$ & 0.25 \\
$\phi_{a}(t)$ & 1 \\
$s(t)$ & $u(t)$ \\
\hline
\end{tabular}

Note that since $f$ is nonlinear in $\omega, \varphi_{\omega}$ is only a linearization. The consistency equation is:

$$
\dot{\hat{x}}_{2}=-\lambda\left(\widehat{x}_{2}-x_{2}\right)+2 \omega_{0} \zeta_{0}\left(1-\mu_{0} x_{1}^{2}\right) x_{2}-w_{0}^{2} x_{1}
$$

The simulation parameters are presented in Table 5 . Note that only small changes in the $\omega$ parameter are to be detected; in this example it will be a $25 \%$ of the maximum change in $\omega_{0}$. The value considered for the additive fault $\phi_{a}(t)$ is also small. $\lambda$ has been chosen rather big in order to reduce $R_{\epsilon}$, and $T$ has been chosen such that several periods of the oscillator output are included in the integration range.

\subsection{Simulation Results}

Figure 1 gives an overview of the behavior of the system, the representative, its mean, and the additive fault before and after the simultaneous fault (these quantities are not affected by the presence of the filter). The state space values do not change significantly due to the fault. Yet, the error function suffers a significant change when the fault occurs. It can be observed that the mean of the representative function is one order of magnitude less than such representative function: this result matches the fact that this mean has been neglected in the theoretical analysis. Note that these representative values are much smaller than the abrupt additive fault function represented in the last plot; this fact supports the validity of the new scheme.

On the other hand, Figure 2 shows the behavior of the estimator for the existing scheme (top figure) and for the new proposed scheme (bottom figure). It is clear that when a simultaneous fault occurs and the old detector/isolator is employed, the estimators do change due to the parametric fault but not enough to get out the upper boundary of the decision region (grey line in the figure). This undesirable situation is not encountered when the new detector/isolator is applied, as it can be seen in the bottom figure; there, the additive fault does not disguise the effect of the parametric one, and the estimators do change beyond the boundary of the decision region, demonstrating the improved behavior of the new proposed scheme.

\section{Conclusions}

A new scheme for the detection and isolation of simultaneous additive and parametric faults in nonlinear stochastic dynamical systems has been presented. A theoretical analysis has been 

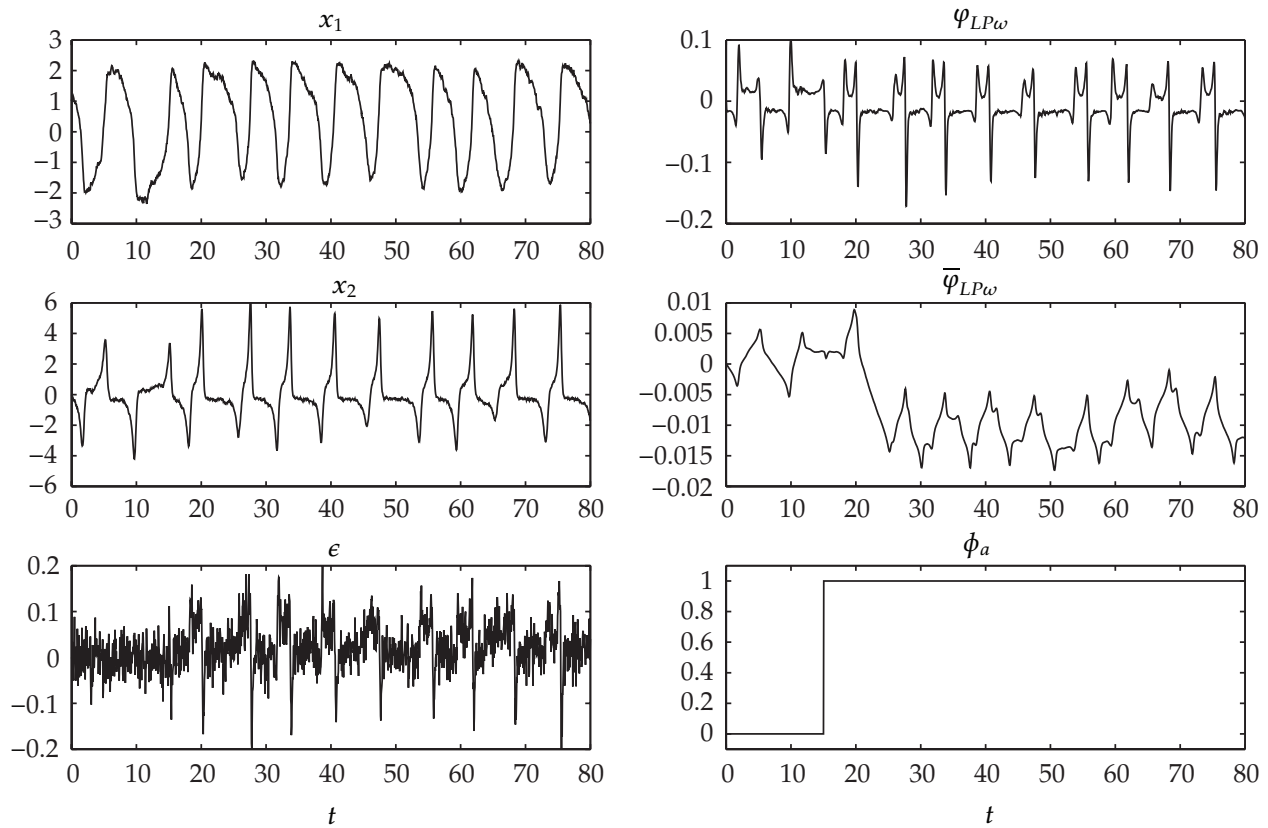

Figure 1: Behavior of the states $x_{1}, x_{2}$, the error function $\epsilon$, the representative $\varphi_{L P \omega}$, its mean $\bar{\varphi}_{L P \omega}$, and the additive fault function $\phi_{a}(t)$ of a simulation with a parameter change in $\omega$ at $T_{0}=15$.
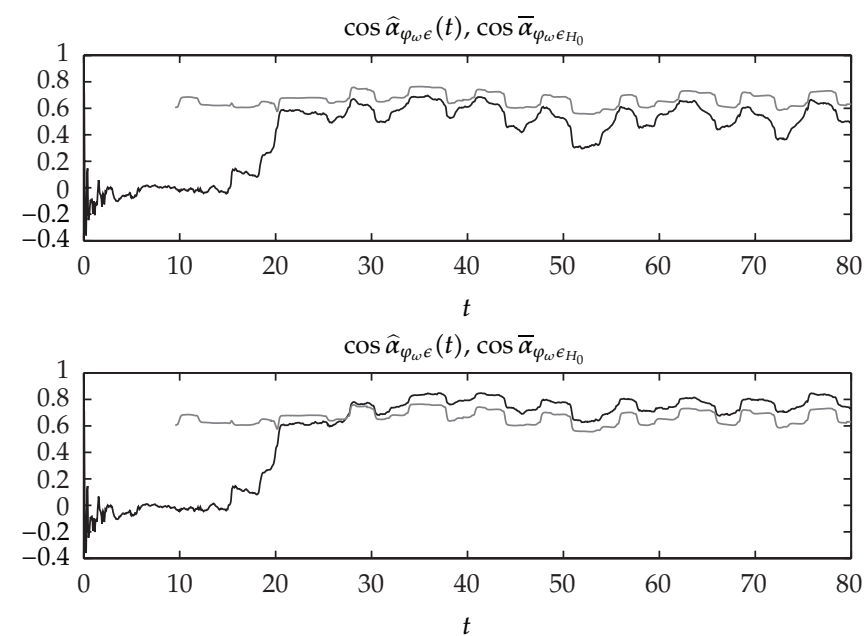

Figure 2: Behavior of the estimator $\cos \widehat{\alpha}_{\varphi_{\omega} \epsilon}(t)$ (black solid line) and the upper boundary $\cos \bar{\alpha}_{\varphi_{\omega} \epsilon_{H_{0}}}$ (grey solid line) of the decision region when a simultaneous fault occurs at $T_{0}=15$ and the old detector/isolator (top figure) or the new proposed detector/isolator is applied (bottom figure).

developed to highlight the limitations of the existing detection/isolation schemes when such types of simultaneous faults occur. Based on the analytical studies, a new detector/isolator has been designed which integrates improved versions of the existing schemes. Comparative simulations have supported the theoretical results by showing the good performance of the new detector/isolator as opposed to the previously existing schemes. 


\section{Acknowledgments}

This work has been partially supported by Project MTM2007-62064 of the Plan Nacional de I+D+i, MEyC, Spain, Project MTM2010-15102 of Ministerio de Ciencia e Innovación, Spain, and by Projects Q09 0930-182 and Q10 0930-144 of the Universidad Politécnica de Madrid (UPM), Spain.

\section{References}

[1] M. Basseville and I. V. Nikiforov, Detection of Abrupt Changes: Theory and Application, Prentice Hall Information and System Sciences Series, Prentice Hall, Englewood Cliffs, NJ, USA, 1993.

[2] J. J. Gertler, Fault Detection and Diagnosis in Engineering Systems, Marcel Dekker, 1998.

[3] R. Iserman, Fault-Diagnosis Systems: An Introduction to Fault Detection and Fault Tolerance, Springer, New York, NY, USA, 2006.

[4] M. M. Polycarpou and A. T. Vemuri, "Learning methodology for failure detection and accommodation," IEEE Control Systems Magazine, vol. 15, no. 3, pp. 16-24, 1995.

[5] G. Biswas, M. O. Cordier, J. Lunze, L. Travé-Massuyès, and M. Staroswiecki, "Diagnosis of complex systems: bridging the methodologies of the FDI and DX communities," IEEE Transactions on Systems, Man, and Cybernetics, Part B, vol. 34, no. 5, pp. 2159-2162, 2004.

[6] P. M. Frank, "Fault diagnosis in dynamic systems using analytical and knowledge-based redundancy. A survey and some new results," Automatica, vol. 26, no. 3, pp. 459-474, 1990.

[7] E. A. García and P. M. Frank, "Deterministic nonlinear observer-based approaches to fault diagnosis: a survey," Control Engineering Practice, vol. 5, no. 5, pp. 663-670, 1997.

[8] P. M. Frank, "Analytical and qualitative model-based fault diagnosis: a survey and some new results," European Journal of Control, vol. 11, no. 2, pp. 26-28, 1996.

[9] C. De Persis and A. Isidori, "A geometric approach to nonlinear fault detection and isolation," IEEE Transactions on Automatic Control, vol. 46, no. 6, pp. 853-865, 2001.

[10] M. M. Polycarpou and A. B. Trunov, "Learning approach to nonlinear fault diagnosis: detectability analysis," IEEE Transactions on Automatic Control, vol. 45, no. 4, pp. 806-812, 2000.

[11] Z. Wang and H. Zhang, "Design of a bilinear fault detection observer for singular bilinear systems," Journal of Control Theory and Applications, vol. 5, no. 1, pp. 28-36, 2007.

[12] X. Zhang, T. Parisini, and M. M. Polycarpou, "Sensor bias fault isolation in a class of nonlinear systems," IEEE Transactions on Automatic Control, vol. 50, no. 3, pp. 370-376, 2005.

[13] B. Jiang, M. Staroswiecki, and V. Cocquempot, "Fault accommodation for nonlinear dynamic systems," IEEE Transactions on Automatic Control, vol. 51, no. 9, pp. 1578-1583, 2006.

[14] M. Du, Fault diagnosis and fault tolerant control of chemical process systems [Ph.D. thesis], McMaster University, 2012.

[15] H. Yang, B. Jiang, V. Cocquempot, and H. Zhang, "Stabilization of switched nonlinear systems with all unstable modes: application to multi-agent systems," IEEE Transactions on Automatic Control, vol. 56, no. 9, pp. 2230-2235, 2011.

[16] X. Zhang, M. M. Polycarpou, and T. Parisini, "Design and analysis of a fault isolation scheme for a class of uncertain nonlinear systems," Annual Reviews in Control, vol. 32, no. 1, pp. 107-121, 2008.

[17] X. Zhang, M. M. Polycarpou, and T. Parisini, “Fault diagnosis of a class of nonlinear uncertain systems with Lipschitz nonlinearities using adaptive estimation," Automatica, vol. 46, no. 2, pp. 290-299, 2010.

[18] M. Basseville, "On-board component fault detection and isolation using the statistical local approach," Automatica, vol. 34, no. 11, pp. 1391-1415, 1998.

[19] S. Tafazoli and X. Sun, "Hybrid system state tracking and fault detection using particle filters," IEEE Transactions on Control Systems Technology, vol. 14, no. 6, pp. 1078-1087, 2006.

[20] Q. Zhang, F. Campillo, F. Cérou, and F. LeGland, "Nonlinear system fault detection and isolation based on bootstrap particle filters," in Proceedings of the 44th IEEE Conference on Decision and Control, and the European Control Conference, (CDC-ECC'05), pp. 3821-3826, December 2005.

[21] A. Xu and Q. Zhang, "Nonlinear system fault diagnosis based on adaptive estimation," Automatica, vol. 40, no. 7, pp. 1181-1193, 2004.

[22] M. W. Hofbaur and B. C. Williams, "Hybrid estimation of complex systems," IEEE Transactions on Systems, Man, and Cybernetics, Part B, vol. 34, no. 5, pp. 2178-2191, 2004.

[23] A. Castillo, Fault detection and isolation via continuous time statistics [Ph.D. thesis], E.T.S. Ingenieros Industriales (Universidad Politcnica de Madrid), 2006. 
[24] A. Castillo and P. J. Zufiria, "Fault detection schemes for continuous-time stochastic dynamical systems," IEEE Transactions on Automatic Control, vol. 54, no. 8, pp. 1820-1836, 2009.

[25] U. Münz and P. J. Zufiria, "Diagnosis of unknown parametric faults in non-linear stochastic dynamical systems," International Journal of Control, vol. 82, no. 4, pp. 603-619, 2009.

[26] P. J. Zufiria, "A formulation for fault detection in stochastic continuous-time dynamical systems," International Journal of Computer Mathematics, vol. 86, no. 10-11, pp. 1778-1797, 2009.

[27] P. J. Zufiria, "Analysis of the detectability time in fault detection schemes for continuous-time systems," in Proceedings of the International Conference Computational and Mathematical Methods in Science and Engineering (CMMSE '09), vol. 4, pp. 1151-1162, Gijón, Spain, 2009.

[28] A. Castillo and P. J. Zufiria, "Fault isolation schemes for a class of continuous-time stochastic dynamical systems," In press.

[29] P. J. Zufiria, "A mathematical framework for new fault detection schemes in nonlinear stochastic continuous-time dynamical systems," Applied Mathematics and Computation, vol. 218, no. 23, pp. 11391-11403, 2012.

[30] B. E. Goodlin, D. S. Boning, H. H. Sawin, and B. M. Wise, "Simultaneous fault detection and classification for semiconductor manufacturing tools," Journal of the Electrochemical Society, vol. 150, no. 12, pp. G778-G784, 2003.

[31] H. Li and J. E. Braun, "A methodology for diagnosing multiple-simultaneous faults in rooftop air conditioners," in Proceedings of the International Refrigeration and Air Conditioning Conference, no. paper 667, 2004, http://docs.lib.purdue.edu/iracc/667.

[32] Y. I. Lu and J. Pond, "Analysis of simutaneous faults using short circuit simulations and fault records," in Proceedings of the Georgia Tech Fault and Disturbance Analysis Conference, May 2008.

[33] I. Yélamos, G. Escudero, M. Graells, and L. Puigjaner, "Simultaneous fault diagnosis in chemical plants using support vector machines," in Proceedings of the 17th European Symposium on Computer Aided Process Engineering (ESCAPE '07), V. Plesu and P. S. Agachi, Eds., Elsevier, 2007.

[34] E. Bullinger and F. Allgower, "An adaptive high-gain observer for nonlinear systems," in Proceedings of the 36th IEEE Conference on Decision and Control, pp. 4348-4353, San Diego, Calif, USA, December 1997.

[35] A. Tornambè, "High-gain observers for nonlinear systems," International Journal of Systems Science, vol. 23, no. 9, pp. 1475-1489, 1992.

[36] M. Reble, U. Münz, and F. Allgöwer, "Diagnosis of parametric faults in multivariable nonlinear systems," in Proceedings of the 46th IEEE Conference on Decision and Control (CDC '07), pp. 366-371, New Orleans, La, USA, December 2007.

[37] A. Papoulis, Probability, Random Variables, and Stochastic Processes, McGraw-Hill, New York, 1965.

[38] X. Zhang, M. M. Polycarpou, and T. Parisini, "A robust detection and isolation scheme for abrupt and incipient faults in nonlinear systems," IEEE Transactions on Automatic Control, vol. 47, no. 4, pp. 576-593, 2002. 


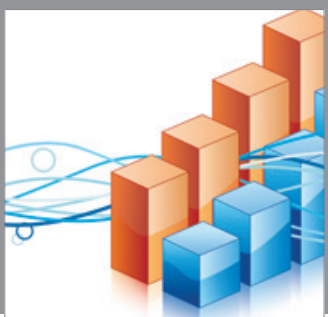

Advances in

Operations Research

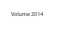

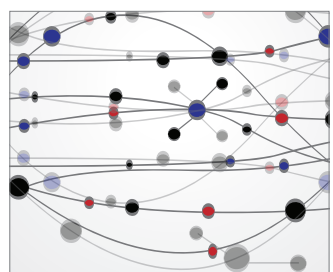

\section{The Scientific} World Journal
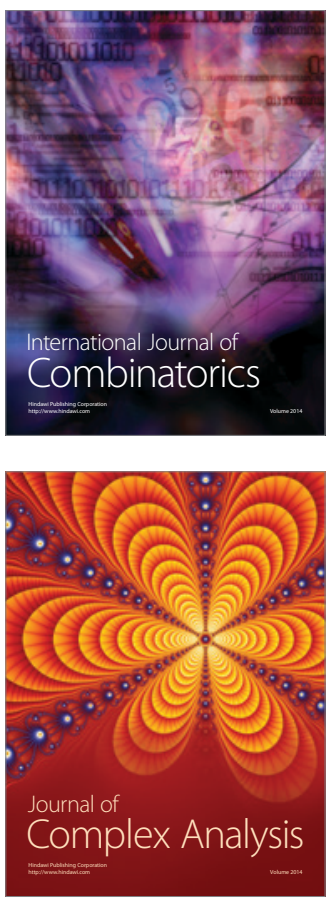

International Journal of

Mathematics and

Mathematical

Sciences


Journal of

Applied Mathematics
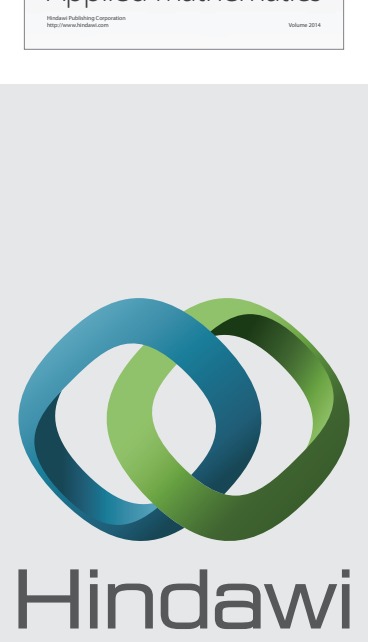

Submit your manuscripts at http://www.hindawi.com


Mathematical Problems in Engineering
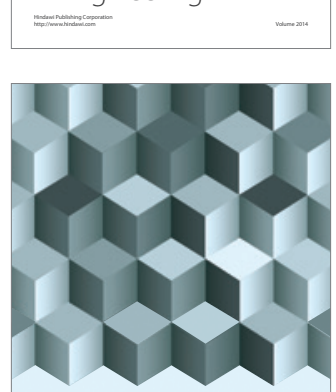

Journal of

Function Spaces
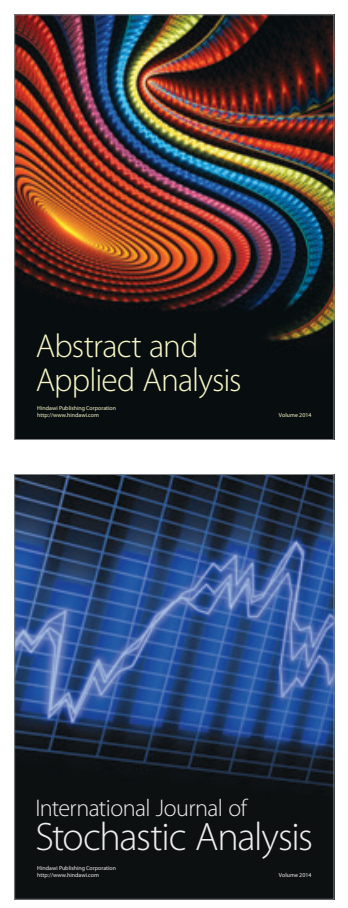

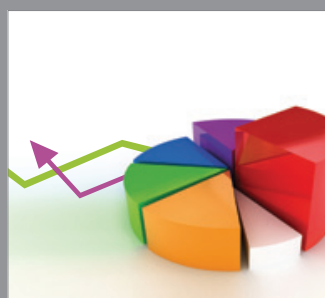

ournal of

Probability and Statistics

Promensencen
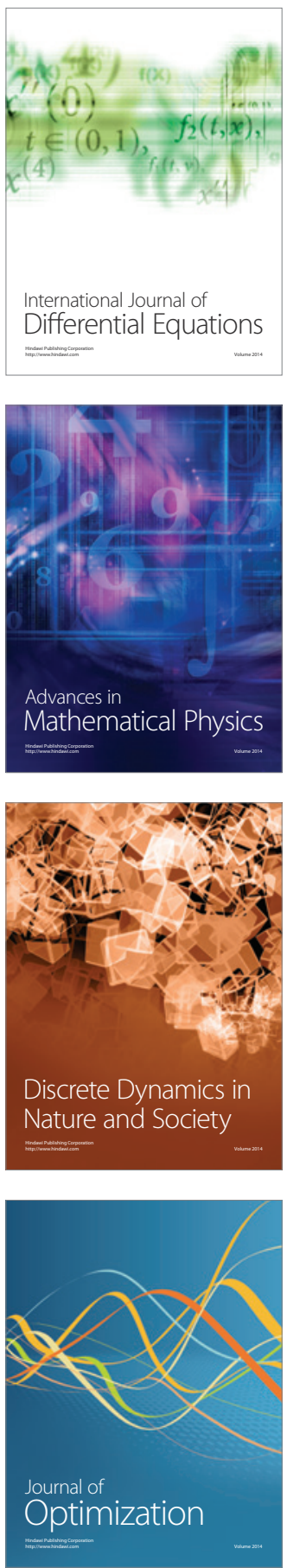